\title{
品|restre
}

\section{A abordagem Design Thinking como proposta de inovação social em um Programa de Visitação Domiciliar na Primeira Infância}

\author{
The Design Thinking approach as a proposal for social innovation in an early Childhood \\ Home Visiting Program
}

\author{
Thaís Pola Baptista Coelho', Lislaine Aparecida Fracolli², Anna Maria Chiesa ${ }^{3}$, Letícia \\ Aparecida da Silva ${ }^{4}$, Hugo Macedo Ferraz e Souza Júnior ${ }^{5}$, Flavio Soares Correa da \\ Silva ${ }^{6}$
}

\section{Resumo}

Objetivo: Utilização da abordagem Design Thinking para melhoria dos protocolos de visitação através da criação de um protótipo escalável, atrativo e que forneça recurso de monitoramento dos resultados do Programa de Visitação Domiciliar "Jovens Mães Cuidadoras". Método: Estudo de desenvolvimento tecnológico por prototipação através da abordagem Design Thinking (DT). Resultados: O protótipo foi criado em papel (cards) e, após a validação pelos participantes do programa, foi transformado em arquivo de texto digital, cuja íntegra está disponível no anexo deste artigo. Conclusão: o público-alvo deste Programa são gestantes adolescentes e crianças que vivem sob alta vulnerabilidade. Se a compreensão por parte dos gestores for padronizada e inflexível quanto à formulação do programa, corre-se o risco de se negligenciar as camadas que formam o ecossistema da criança atendida. Na melhor das hipóteses, pode-se projetar soluções ou intervenções que tenham um impacto social moderado; e, na pior das hipóteses, é possível que o programa, além de não ter eficácia, reforce as injustiças e a desigualdade social. Por esta razão, recomenda-se que qualquer programa ou serviço de impacto social priorize o desenho de escopo de forma a se adaptar ao contexto de sua aplicação.

Palavras-Chave: Inovação Social. Visita domiciliar. Primeira Infância.

\section{Abstract}

Objective: Application of Design Thinking to improve visitation protocols by creating a scalable and attractive prototype that provides results monitoring of the "Young Mother Caregiver" Home Visiting Program. Methodology: Technological development study by

\footnotetext{
1 Mestre em Ciências. Universidade de São Paulo - Escola de Enfermagem. Email: thaispola@hotmail.com 2 Doutora, professora associada. Universidade de São Paulo - Escola de Enfermagem. Email: lislaine@usp.br 3 Doutora, professora associada sênior. Universidade de São Paulo - Escola de Enfermagem. Email: amchiesa@usp.br 4 Mestranda. Universidade de São Paulo - Escola de enfermagem. Email: silvaleticia@gmail.com

5 Doutor, professor. Faculdade de Medicina do ABC - Departamento de Saúde da Coletividade. Email: hugomacedojr@hotmail.com

6 Doutor, professor associado. Universidade de São Paulo - Instituto de Matemática e Estatística. Email: correadasilva_f2001@yahoo.com.br

Correspondência: Av. Dr. Enéas Carvalho de Aguiar, 419 - Cerqueira César, São Paulo - SP - Brasil. CEP: 05403-000
}

RE. SAÚD. DIGI. TEC. EDU., Fortaleza, CE, v.4, n.2, p.08-19, ago./dez. 2019.

ISSN: 2525-9063 
prototyping using Design Thinking (DT). Results: The prototype was created on paper (cards) and, once validated by the program participants, was transformed into a digital text file, which is fully available in the appendix to this article. Conclusion: The target audience of this Program are teenage pregnant women and children living under high vulnerability. If managers' understanding become standardized and inflexible about the program design, there is a risk of neglecting the layers that compose the ecosystem of the assisted child. At best, solutions or interventions with a moderate social impact can be designed; and at worst, the program may not only be ineffective, but also reinforce injustice and social inequality. Hence, it is recommended that any social impact program or service prioritize scope design in order to become tailored to the context of its application.

Keywords: Social Inovattion. Home visit. Childhood.

\section{Introdução}

Os primeiros 1000 dias de vida representam uma janela de oportunidade de estimulação para que a criança possa atingir o seu potencial máximo de crescimento e desenvolvimento na vida adulta. Cuidados precários de saúde, desnutrição, estimulação inadequada e estressores ambientais nessa janela de tempo que compreende as 40 semanas de gestação (280 dias) somadas aos 2 primeiros anos de vida (730 dias), comprometem o desenvolvimento infantil e aumenta a chance de que o potencial dos indivíduos não seja alcançado ao longo da vida ${ }^{1}$. Intervenções para melhorar os cuidados de saúde, as competências pessoais de mães vulneráveis, começando durante a gravidez, são estratégias promissoras para proteger o cérebro em desenvolvimento de seus filhos e sua própria saúde física e mental ${ }^{2,3,4}$. Estas intervenções parecem ser particularmente importantes para pais com história de adversidades no início de suas vidas, não tendo com isso oportunidades de desenvolver as habilidades necessárias para criar um ambiente propício para que suas crianças desenvolvam suas próprias capacidades adaptativas ${ }^{3,5}$.

Embora este cenário esteja presente mundialmente, ele ocorre principalmente em países com predomínio de populações de baixa renda, como o Brasil. Mais de 200 milhões de crianças menores de cinco anos nestes países não atingem o seu potencial de desenvolvimento por causa da insuficiência e da baixa qualidade da estimulação que seus pais conseguem oferecer ${ }^{6}$. Em países desenvolvidos, a valorização das relações precoces entre mãe-bebê tem sido um foco importante dos gestores públicos nos últimos 30 anos ${ }^{1}$.

Baseado em análises objetivas de custo-benefício, os programas de visitas domiciliares com foco no aprimoramento das relações mãe-bebê têm recebido grande atenção. Estes programas são considerados uma ferramenta importante para melhorar a saúde e o desenvolvimento da criança e, para isso tem por objetivo melhorar, além da saúde 
da mãe no pré-natal, as ferramentas que os pais possuem para cuidar e estimular seu bebê adequadamente ${ }^{7,8}$

Desafios sociais requerem soluções sistêmicas baseadas nas necessidades de seus usuários portanto exigem uma abordagem centrada nas pessoas para a identificação coletiva dos problemas e resolução compartilhada, não apenas de produtos, como também serviços ou programas sociais ${ }^{9}$.

Na última década o Design Thinking (DT) vem sendo utilizado como um meio de desenvolver inovações nas organizações. Esta abordagem envolve o processo de geração de ideias em um grupo multidisciplinar como foco na resolução de problemas, assim, a utilização do design vai além da aparência dos produtos, sendo aplicado na concepção de soluções, que englobam os aspectos estratégicos de projetos e/ou negócios ${ }^{10}$.

A abordagem Design Thinking (DT) teve origem em 1991, nos Estados Unidos pela empresa de inovação e design global IDEO, com o foco de melhoria em de produtos entre eles a escova de dente Oral B, o IPod da Apple e a cadeira Aeron da Herman Miller- sob a seguinte premissa: se você projeta uma cadeira, projete-a para a pessoa que estará sentada nela oito horas por dia 9 .

Atualmente vem sendo incorporado na área da saúde em centros hospitalares, numa publicação da Harvard Business Review destaca a transformação de um Hospital de Olhos de Roterdã na Holanda.
Segundo os autores o objetivo do Design Thinking neste caso, foi criar uma estrutura que tornasse todos os envolvidos responsáveis pelos resultados. $\mathrm{A}$ área da saúde é um ecossistema complexo, tem muitas pessoas envolvidas. Nesta esfera deve-se considerar o usuário/paciente, os familiares, os profissionais diretamente ligados ao cuidado (recepcionistas, técnicos, enfermeiros, médicos e equipe multiprofissional), além dos profissionais que dão suporte a estrutura física e administrativa. Na maioria dos casos, o serviço é orientado pelo hospital, pensando no que gera menor custo e o que é mais viável. O que acontece, atualmente, é essa revolução de pensar mais no usuário. É preciso, por exemplo, repensar a espera na emergência, a falta de uma triagem com processos mais rápidos ${ }^{12}$.

Por este motivo, a área da saúde possui uma grande demanda por inovação, as soluções de melhoria devem reconhecer a experiência de todas as pessoas envolvidas no processo de cuidado. Desde os usuários/pacientes aos recepcionistas, enfermeiros e médicos. O usuário deve ser pensado em todas as etapas. "Como é o contato do usuário com o serviço? Isso vai desde o momento que ele sente a necessidade e se desloca para o hospital, até o momento que a sua demanda é avaliada e atendida. A saúde tem muitas possibilidades para o design, mas exige uma visão de viabilidade para que haja equilíbrio entre a demanda do 
usuário e a capacidade de resposta da equipe ${ }^{12}$.

Mais recentemente afirmam Bernstein \& Lisnky, que os praticantes do DT começaram a aplicar esse método para melhoria de serviços públicos - como aqueles destinados aos cuidados em saúde de baixo custo e melhorias organizacionais de centros de educação como a criação de escolas públicas melhores ${ }^{9}$.

O design é algo novo em todas as áreas, não apenas na saúde. O ideal é que seja implantado por gestores, administradores, mas há a possibilidade de começar a ser utilizado aos poucos, com os próprios profissionais analisando oportunidades de melhorar produtos e serviços. A inovação guiada pelo design tem chamado atenção do mundo todo, do universo de negócios à área social, devido aos seus processos abertos e colaborativos para o desenvolvimento de projetos $^{12}$.

O design não é apenas estético, é um modelo mental, uma forma de pensar para resolver problemas complexos ${ }^{12}$. O setor social tem se envolvido com a mentalidade e os métodos ágeis de projetos centrados no ser humano, com o foco de melhorar vidas ao invés da melhoria de produtos ${ }^{13}$.

Entretanto até o presente momento não há qualquer publicação que evidencie a utilização da abordagem DT para a melhoria de programas de visitação domiciliar voltada a primeira infância no
Brasil, o que demonstra a natureza inovadora deste artigo.

Diante deste cenário, no que diz respeito a operacionalização e a escalabilidade do Programa Jovens Mães Cuidadoras, foi proposta a abordagem Design Thinking para melhoria dos protocolos de visitas de forma a se estabelecer um protótipo escalável, atrativo que forneçam recursos de monitoramento dos resultados do programa.

\section{Métodos}

Este é um estudo de desenvolvimento tecnológico por prototipação através da abordagem Design Thinking (DT). O DT não é uma metodologia e sim uma abordagem. Isso porque quando se pensa em método se cria uma expectativa de uma fórmula matemática que se aplique indistintamente em qualquer situação, o inverso do que se espera neste caso.

O processo consiste em tentar mapear e mesclar a experiência cultural, a visão de mundo e os processos inseridos na vida dos indivíduos, no intuito de obter uma visão mais completa na solução de problemas e, dessa forma, melhor identificar as barreiras e gerar alternativas viáveis para transpô-las.

Os sujeitos do estudo foram as partes envolvidas diretamente com 0 Programa Jovens Mães Cuidadoras, ou seja, as Enfermeiras Visitadoras, a 
Gestora PJMC e a Supervisora Clínica, conforme figura 1 em anexo.

As etapas do DT são 5 e devem ser realizadas de maneira cíclica pois combinam o percurso do pensamento (reconhecimento do problema e clareza das ideias de enfrentamento) com a sistematização da gestão de um projeto, são elas: 1) Descoberta, 2) Imersão, 3) Ideação, 4) Experimentação e 5) Evolução.

\section{Resultados}

\section{DESCOBERTA - FASE 1}

A primeira fase consiste na observação das pessoas envolvidas no processo para que se possa compreender seus pain points (pontos de dor) antes de se começar a pensar no projeto. Este momento foi realizado na escola de enfermagem com entrevistas da pesquisadora com os stakeholders principais: enfermeira visitadora, gestora e supervisora clínica, conforme informações da Tabela 1 abaixo.

\begin{tabular}{|c|c|}
\hline \multicolumn{2}{|c|}{$\begin{array}{c}\text { Tabela 1: Descoberta - Identificação dos } \\
\text { pontos de dor pain points dos } \\
\text { stakeholders do PJMC }\end{array}$} \\
\hline 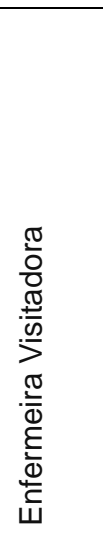 & $\begin{array}{l}\text { Dificuldade de acessar as } \\
\text { informações relevantes nas } \\
\text { visitas passadas. Falta de } \\
\text { espaço para escrever o } \\
\text { lembrete para a próxima visita. } \\
\text { Tomada de decisão da } \\
\text { enfermeira mais focada na } \\
\text { fotografia do momento do que } \\
\text { na evolução e desempenho da } \\
\text { gestante no decorrer do PJMC. } \\
\text { Excesso de material para } \\
\text { transportar (cadernos e } \\
\text { manuais técnicos). }\end{array}$ \\
\hline 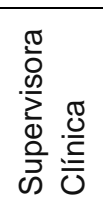 & $\begin{array}{l}\text { Não tem informações de } \\
\text { desempenho da gestante e } \\
\text { pontos de atenção de uma } \\
\text { forma simplificada para a } \\
\text { discussão clínica. }\end{array}$ \\
\hline 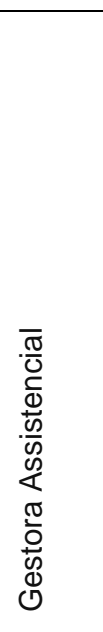 & $\begin{array}{l}\text { O PJMC prescinde de } \\
\text { identidade visual e organização } \\
\text { padrão e afetam a sua } \\
\text { escalabilidade. } \\
\text { Como gestora não consigo } \\
\text { acompanhar a evolução da } \\
\text { assistência a não ser pela } \\
\text { leitura na íntegra do protocolo } \\
\text { ou pelo relato da enfermeira. } \\
\text { Não consegue saber se a visita } \\
\text { foi boa ou ruim se não } \\
\text { perguntar a enfermeira. E não } \\
\text { tem uma maneira de conhecer } \\
\text { os imprevistos e propor estudos } \\
\text { sentinelas. }\end{array}$ \\
\hline
\end{tabular}

Fonte: Imagem extraída da dissertação COELHO, TPB, 2018.

\section{IMERSÃO - FASE 2}

Esta etapa tem o objetivo de captar percepções (insights), compartilhar anotações e registros de pensamento, observações e histórias e também foi realizado na escola de enfermagem com entrevistas da pesquisadora com os stakeholders principais: enfermeira, gestora e supervisora do programa. 
IDEAÇÃO - FASE 3

Este é o momento conhecido como brainstorming, onde acontece a geração de ideias em que todos os participantes apresentam suas ideias por meio de palavras ou desenhos sem preocupação com a seleção, o importante é deixar fluir desejos e possibilidades. Foram gravadas as entrevistas e os desejos dos stakeholders envolvidos e transcritos como histórias em papel.

\begin{tabular}{|c|c|}
\hline \multicolumn{2}{|c|}{$\begin{array}{c}\text { Tabela } 2 \text { - Ideação - Brainstorming } \\
\text { dos stakeholders do PJMC }\end{array}$} \\
\hline 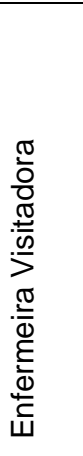 & $\begin{array}{l}\text { Que o novo material possa facilitar a } \\
\text { preparação para a visita, mostrando } \\
\text { o material a ser utilizado de uma } \\
\text { forma resumida. } \\
\text { Que tenha espaço para evoluir os } \\
\text { pontos principais a serem } \\
\text { trabalhados ou retomados no } \\
\text { momento da visita. } \\
\text { Que reúna a maioria dos materiais } \\
\text { impressos na intervenção para não } \\
\text { carregar muito peso. }\end{array}$ \\
\hline 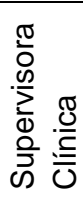 & $\begin{array}{l}\text { Que tenha um campo de evolução do } \\
\text { desempenho da gestante e dos } \\
\text { pontos críticos para discutir na } \\
\text { supervisão clínica de maneira } \\
\text { resumida e rápida. }\end{array}$ \\
\hline 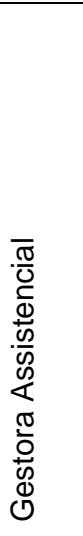 & $\begin{array}{l}\text { Ter um aplicativo que apresente uma } \\
\text { videoteca ou um material impresso } \\
\text { que indique os vídeos a serem } \\
\text { baixados. } \\
\text { Ter um campo quantitativo com } \\
\text { estatísticas da evolução das } \\
\text { gestantes no programa. } \\
\text { Ter o monitoramento das visitas } \\
\text { realizadas e não realizadas no PJMC } \\
\text { para avaliação da macrogestão e na } \\
\text { supervisão clínica. } \\
\text { Visão geral dos resultados das } \\
\text { escalas aplicadas. }\end{array}$ \\
\hline
\end{tabular}

Fonte: Imagem extraída da dissertação COELHO, TPB, 2018.
EXPERIMENTAÇÃO - FASE 4

A fase de dar vida as ideias. Criar protótipos para tornar as ideias tangíveis e depois apresentar as pessoas para que se possa analisar e refinar a compreensão coletiva.

\begin{tabular}{|c|l|}
\hline \multicolumn{1}{|l|}{ Tabela 3 - Protótipo do protocolo do } \\
PJMC: fase de construção dos \\
requisitos de negócio
\end{tabular}




\begin{tabular}{|c|c|}
\hline 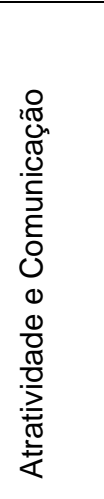 & $\begin{array}{l}\text { Que seja mais atrativo que o protocolo } \\
\text { atual; } \\
\text { Que o protocolo atual seja passível de } \\
\text { atualização/adaptação de acordo com } \\
\text { a realidade do PJMC e seja intuitivo e } \\
\text { amigável para as enfermeiras } \\
\text { visitadoras; } \\
\text { Que as cores e a interface do protótipo } \\
\text { favoreçam a parentalidade; } \\
\text { Que as informações importantes } \\
\text { sejam inseridas como lembrete na } \\
\text { preparação da visita. }\end{array}$ \\
\hline 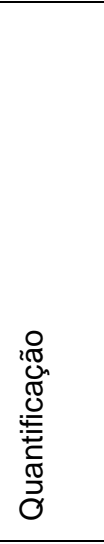 & $\begin{array}{l}\text { Que seja possível mensurar os dados } \\
\text { com o resultado das intervenções } \\
\text { realizadas de uma forma fácil e } \\
\text { replicável; } \\
\text { Que armazene os registros de quantas } \\
\text { gestantes e famílias estão em } \\
\text { acompanhamento; } \\
\text { Que possibilite uma avaliação ainda } \\
\text { que subjetiva (carinhas) do } \\
\text { profissional paciente em cada visita; } \\
\text { Que possibilite uma avaliação dos } \\
\text { macro resultados do programa de uma } \\
\text { forma analítica e quantificável. }\end{array}$ \\
\hline 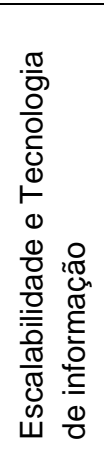 & $\begin{array}{l}\text { Que o protocolo seja padronizado de } \\
\text { modo que sua especificação e } \\
\text { programação seja facilitada no futuro; } \\
\text { Que seja futuramente transformado } \\
\text { num app compatível com o sistema } \\
\text { operacional Android (tablets); } \\
\text { Que futuramente o aplicativo possa } \\
\text { funcionar "offline" ao se } \\
\text { operacionalizar na visita e possa ser } \\
\text { atualizado em rede wifi ou 4G } \\
\text { posteriormente. }\end{array}$ \\
\hline
\end{tabular}

Fonte: Imagem extraída da dissertação COELHO, TPB, 2018.

\section{EVOLUÇÃO - FASE 5}

Uma vez criado o produto, é importante planejar os passos futuros para que ele seja realizado, acompanhado e avaliado. A construção e aprendizado são permanentes. O protótipo foi criado em papel pela pesquisadora em forma de CARDS (cartões por assunto) e após a apresentação e aprovação dos stakeholders foi transformado em arquivo digital, podendo ser consultado em sua íntegra no anexo 2 e as principais criações e modificações também foram inseridas na tabela 4 , ambos no final deste artigo por sua extensão.

\section{Conclusão}

O desafio posto para
implementadores de políticas ou programas sociais consiste na inovação da maneira de pensar e planejar os processos de cuidado e os programas na saúde. É preciso ter clareza de onde se quer chegar e principalmente a quem se destina a intervenção, antes de padronizar e sistematizar o cuidado.

Comunicar, registrar e gerir os resultados em saúde é um desafio cotidiano que se torna imprescindível diante do subfinanciamento do Sistema Único de Saúde. Esta escassez de recursos nos convoca a uma gestão mais criativa e eficiente dos programas existentes. Repensar os programas e o cuidado em saúde sob uma lógica digital pode uma estratégia de eficácia e inovação em saúde. A tecnologia digital em síntese representa 0 acesso rápido à informação. Adotar a mentalidade digital implica em assumir uma atitude de constante construção, padronização e renovação. É preciso ter a percepção de como as novidades que nos permeiam criarão impactos no futuro. Pensar digitalmente não é somente ter acesso às informações da rede eletrônica ou de um aplicativo, mas também ser capaz de 
utilizar o conteúdo destes e transformá-los em um valor ou benefício.

No setor social ou se saúde não se trata de desenvolver um produto, mas sim de melhorar vidas. Além do que, as vidas humanas são inerentemente complexas e inseridas em contextos únicos que moldam cada passo que damos e cada decisão que tomamos. Portanto, para projetar um programa com eficácia, é preciso analisar e levar em conta o contexto em que as vidas são conduzidas. Para além das etapas e preceitos adotados no Design Thinking, é preciso adaptar os mesmos em situações que envolvam o setor social, para que se alcance em essência 0 contexto de maneira profunda.

No caso do Programa Jovens Mães Cuidadoras, as gestantes são adolescentes e vivem sob alta vulnerabilidade. Se a compreensão por parte dos gestores for padronizada e inflexível quanto a formulação do programa, corre-se o risco de negligenciar as camadas que formam o ecossistema das crianças atendidas, na melhor das hipóteses, pode-se projetar soluções ou intervenções que tenham um impacto moderado na população atendida; e na pior das hipóteses, é possível que o programa além de não ter eficácia reforce as injustiças e desigualdades que a mãe e a criança estão submetidas.

Por fim recomenda-se que programas ou serviços de impacto social devem ser submetidos a redesenhos de escopo de forma contínua e cooperativa. É necessário sentar lado a lado com o público atendido e demonstrar total consideração ao seu contexto atual, pois a vida é dinâmica e exige um processo onde se aprende, idealiza, testa e se adapta.

A tecnologia não resolve os problemas que não são pensados com a nossa própria cabeça. Um registro digital em saúde exige o conhecimento de múltiplos conceitos envolvidos no processo de entender o que se quer com um diagnóstico antes de se diagnosticar. Por este motivo as necessidades relacionadas à assistência e à gestão do PJMC foram amplamente exploradas antes de produzir a nova versão do instrumento de trabalho de trabalho das enfermeiras visitadoras, até o momento em que se identifique novas soluções para as necessidades encontradas e este ciclo cooperativo recomece.

\section{Agradecimentos}

A professora Lislaine Fracolli por me apresentar o universo da primeira infância.

A professora Anna Chiesa por me encorajar a assumir o desafio de cursar o Curso Executivo em Liderança em Política Pública em Primeira Infância em Harvard, que me iluminou para produção deste artigo.

Ao meu esposo Ediléu Cardoso, que sempre me incentiva na busca de conhecimento e me apresenta ao universo da inovação. 
Conflito de interesse: Este artigo é desprovido de qualquer conflito de interesse ou interesse pessoal/comercial.

\section{Referências}

1. Coelho TPB. O raciocínio da enfermagem na era digital: uma versão renovada do protocolo de intervenção do Programa Jovens Mães Cuidadoras. São Paulo. Dissertação [Mestrado em Ciências da Saúde]. - Escola de Enfermagem da Universidade de São Paulo; 2018. IN:

http://www.teses.usp.br/teses/disponiveis/ 7/7144/tde-05112018-123324/pt-br.php

2. Shonkoff JP et al An Integrated Scientific Framework for Child Survival and Early Childhood Development.

Pediatrics; 129 (2): 1-13; 2012.

3. Shonkoff JP. Building a new biodevelopmental framework to guide the future of early childhood policy. Child Dev ;81(1):357-67; 2010.

4. Shonkoff JP. Protecting brains, not simply stimulating minds.

Science;333(6045):982-984; 2010.

5. Kieling $\mathrm{C}$, Baker-Henningham $\mathrm{H}$, Belfer M, Conti G, Ertem I, Omigbodun O, Rohde LA, Srinath S, Ulkuer N, Rahman A Child and adolescent mental health worldwide: evidence for action. The Lancet ;378(9801):1515-25; 2011.

6. Olds DL, Sadler L, Kitzman HJ. Programs for parents of infants and toddlers: recent evidence from randomized trials. Child Psychol Psychiatry;48(3-4):355-91. Review; 2007. 7. Kahan J \& Moore KA What. Works for Home visiting programs: lesson from experimental evaluations of program and interventions. Child Trends; 1-33; 2010. 8. Donelan-McCall N, Eckenrode J, Olds DL. Home visiting for the prevention of child maltreatment: lessons learned during the past 20 years. PediatrClin North Am; 56(2):389-403; 2009.

9. Brown, T, Wyatt J. Design Thinking for Social Innovation. Stanford Social Innovation Review. 31-35; 2010. 10. Macedo M, Miguel PA, Casarotto Filho N. A caracterização do Design
Thinking como um modelo de inovação. Inmr, rev. adm. inov. [Internet]. 29set.2015 [citado 20nov.2019];12(3):157-82.

Available from:

http://www.revistas.usp.br/rai/article/view/ 101357

11. Bernstein M, Lisnky, M. Leading Change Trough Adaptative Design.

Stanford Social Innovation Review. 16-27; 2015

12. Deichmann D, Heijde Rvd. How Design Thinking Turned One Hospital into a Bright and Comforting Place. Harvard Bussiness Review, 2016. https://hbr.org/2016/12/how-designthinking-turned-one-hospital-into-a-brightand-comforting-place

13. Martin, $\mathrm{R}$. The design of business: Why design thinking is the next competitive advantage. Boston: Harvard Bussiness Press, 2009. 


\section{Anexos}

Figura 1: Apresentação dos stakeholders do Programa Jovens Mães Cuidadoras.

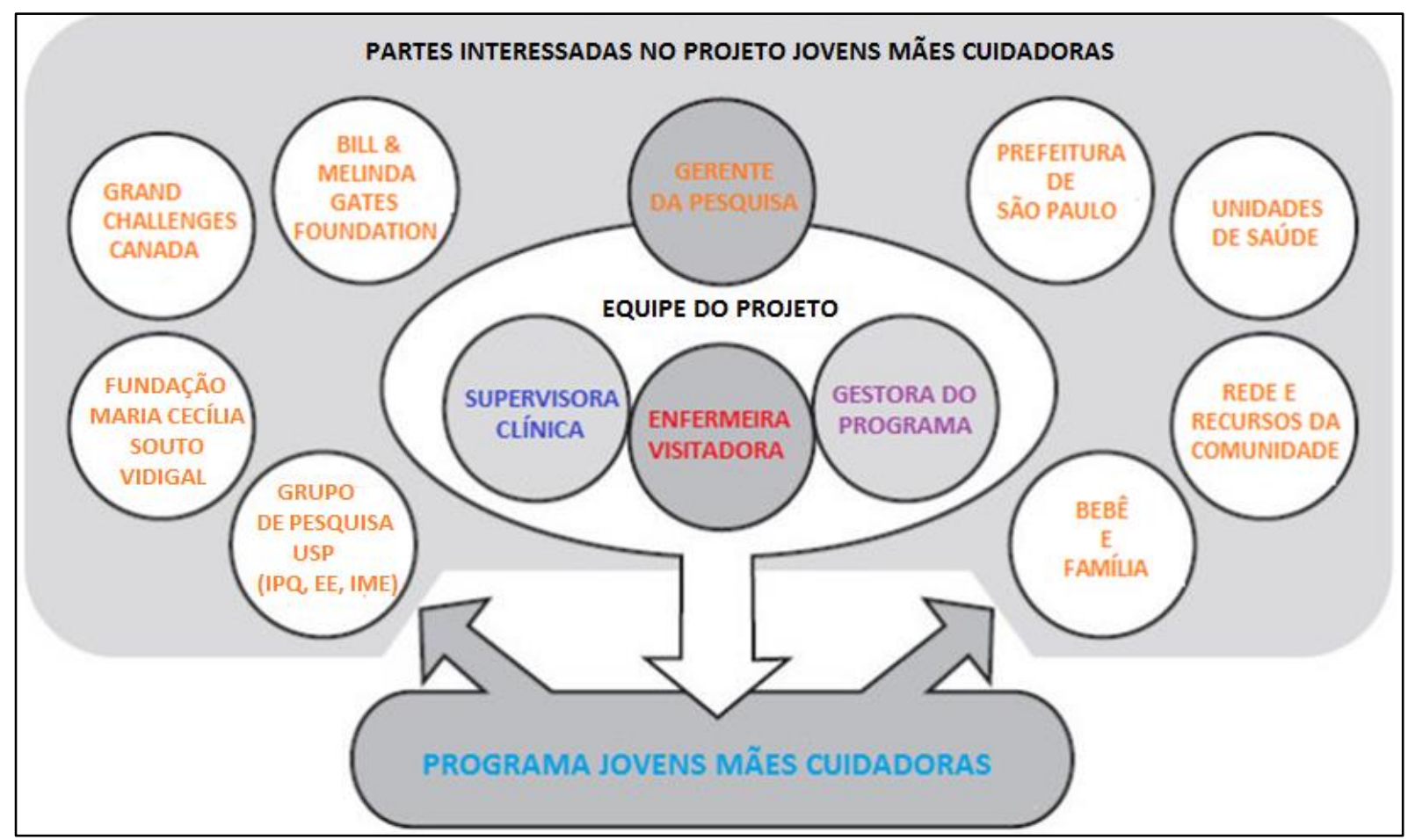

Fonte: Imagem extraída da dissertação Coelho, TPB, 2018, de sua própria autoria.

Anexo 2: Protocolo de Visita de Intervenção

http://www.ee.usp.br/posgraduacao/mestrado/apostilas/Protocolo.pdf

RE. SAÚD. DIGI. TEC. EDU., Fortaleza, CE, v.4, n.2, p.08-19, ago./dez. 2019.

ISSN: 2525-9063 
Tabela 4 - Protótipo final -Principais pontos de formatação do novo protocolo de visitas de intervenção

Atratividade
Inserção de ícones
com padrão de
orientação e
organização em
seções. Construção
do tutorial de
utilização do

protocolo renovado

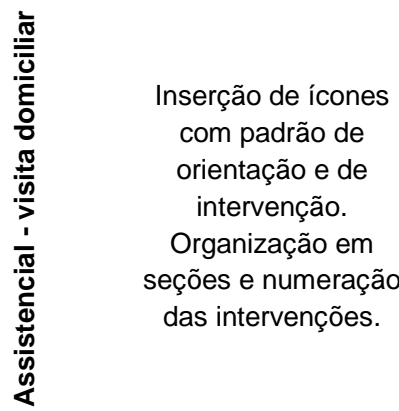

\section{Organização Escalabilidade}

Formatação do cadastro e cabeçalho da visita.

Reorganização do

roteiro de preparação de visita.

\section{Padronização das}

instruções por

intervenção, material

didático e instrumento de apoio.
Fase de instrução não exige quantificação

\begin{abstract}
Sistematização das intervenções de acordo com as premissas do programa (Cuidados com a Saúde, Saúde Ambiental, Curso de Vida, Família, Rede Social, Parenting) Criação do campo de intervenção da Visita Domiciliar.
\end{abstract}

Inclusão das instruções

o que e como no cabeçalho das intervenções.
Construção de campos quantificáveis: (check-list) booleanos, combo, listas. Redução dos campos de texto ou string que não permite a quantificação.
Inclusão da fase final: 0 resultado é o produto final da intervenção realizada na visita.
Criação de um padrão de avaliação (fórmula) que prioriza o parenting e o cuidado em saúde $e$ contempla as demais premissas do PJMC

Construção do Diagnóstico Geral da Gestante no PJMC;

Criação da ficha de resultado e Desempenho Final da Gestante no programa PJMC.

Fonte: Imagem extraída da dissertação Coelho, TPB, 2018. 


\section{Como citar este artigo}

Coelho TPB, Fracolli LA, Chiesa A, Silva L, Ferraz HMS, Silva FSC. A abordagem Design Thinking como proposta de inovação social em um Programa de Visitação Domiciliar na Primeira Infância. Revista de Saúde Digital e Tecnologias Educacionais. [online], volume 4, n. 2. Editor responsável: Luiz Roberto de Oliveira. Fortaleza, mês e ano, p. 08-19. Disponível em: http://periodicos.ufc.br/resdite/index. Acesso em "dia/mês/ano".

Data de recebimento do artigo: 22/09/2019

Data de aprovação do artigo: 09/11/2019 